\title{
The RICH detector of the NA62 experiment at CERN
}

\author{
Monica Pepe ${ }^{1}$ \\ INFN Perugia \\ Via A. Pascoli, 06123 Perugia, Italy \\ E-mail: monica.pepe@pg.infn.it
}

\section{Patrizia Cenci}

INFN Perugia

Via A. Pascoli, 06123 Perugia, Italy

E-mail: patrizia.cenci@pg.infn.it

The NA62 experiment at CERN aims at the measurement of the ultra-rare decay $\mathrm{K}^{+} \rightarrow \pi^{+} v \bar{v}$ with $10 \%$ accuracy. This requires the suppression of the main background channel $\mathrm{K}^{+} \rightarrow \mu^{+} v$ that can be achieved through kinematical cuts and featuring the different stopping power of $\mu^{+}$and $\pi^{+}$through matter. A further $\mu^{+}$suppression in a momentum range between 15 and $35 \mathrm{GeV} / \mathrm{c}$ is obtained by means of a RICH detector filled with Neon at atmospheric pressure, contained in a $17 \mathrm{~m}$ long, $3 \mathrm{~m}$ wide vessel and readout by about 2000 single-anode photomultipliers. The main features of the RICH detector of NA62 are shown and the results of test beams performed at the CERN SPS with a prototype are presented.

2009 KAON International Conference KAON09

Tsukuba, Japan

June 9-12, 200

\footnotetext{
$1 \quad$ Speaker
} 


\section{Introduction}

The NA62 experiment at CERN [1] aims at the measurement of the ultra-rare process $\mathrm{K}^{+} \rightarrow \pi^{+} v \bar{v}$ with a $10 \%$ accuracy. According to the Standard Model (SM), the branching fraction of this mode is $\mathrm{BR}=(0.85 \pm 0.07) \times 10^{-10}[2-5]$. The most recent measurement, based on 7 events observed by the E787 and E959 Collaborations at BNL, is BR $=\left(1.73_{-1.05}^{+1.15}\right) \times 10^{-10}[6]$. Sizable deviations from SM are predicted in a variety of models [7-8].

In order to fulfil the NA62 purpose, a background over signal ratio smaller than $10 \%$ should be reached. This requires a suppression factor of about $4 \times 10^{-13}$ of the main background process $\mathrm{K}^{+} \rightarrow \mu^{+} v$, achieved by combining different methods.

According to the Monte Carlo simulation of the experiment, a suppression of $8 \times 10^{-6} \mathrm{can}$ be obtained through kinematical cuts. A muon rejection factor of $10^{-5}$ can be achieved exploiting the different stopping power of muons and pions through matter. A further $5 \times 10^{-3}$ pion to muon suppression factor is provided by a Ring Imaging Cherenkov (RICH) detector, in a momentum range between 15 and $35 \mathrm{GeV} / \mathrm{c}$.

The RICH proposed for the NA62 experiment is filled with Neon gas at atmospheric pressure, contained in a $17 \mathrm{~m}$ long, $3 \mathrm{~m}$ wide vessel. The Cherenkov light is read by about 2000 single-anode fast photomultipliers.

\section{The RICH detector design}

In a RICH detector [9] the Cherenkov light, emitted at an angle $\theta_{c}$ by a charged particle of velocity $\beta c$, larger than the speed of light in the crossed medium $(c / n)$, is imaged by means of a spherical mirror onto a ring on its focal plane. For small $n$, as in case of gas radiators, the ring radius $r$ is related to the Cherenkov angle as $\theta_{c}=r / f$, where $f$ is the mirror focal length.

In order to achieve the required $\pi-\mu$ separation, the NA62 RICH must have a Cherenkov angle $\theta_{c}$ resolution better than $80 \mu \mathrm{rad}$. Moreover, it must provide the crossing time of the pion produced in the $\mathrm{K}^{+}$decay with a resolution of $100 \mathrm{ps}$, useful to suppress accidental coincidences with an upstream beam detector. Finally, it should give the level-zero fast trigger for a charged particle. The latter requirements led to the choice of small size photomultipliers.

The NA62 RICH must identify pions and muons with momenta between 15 and $35 \mathrm{GeV}$. The best $\pi-\mu$ separation is obtained when the lowest accepted momentum is close to the Cherenkov threshold. However, full efficiency is achieved at momenta about $20 \%$ higher than the threshold. For the above reason, the Cherenkov threshold should be $12.5 \mathrm{GeV} / \mathrm{c}$ for a pion, i.e. the index of refraction $n$ must be given by $(n-1) \approx 60 \times 10^{-6}$.

Neon gas at atmospheric pressure fulfils this requirement; it also guarantees a small dispersion [10]. However, the smallness of $(n-1)$ implies a low emission of Cherenkov photons per unit length which should be compensated with a long radiator. The NA62 RICH will make use of the maximum space available along the beam line, i.e. about $18 \mathrm{~m}$. Therefore, a stainless steel cylindrical vessel is foreseen, about $3 \mathrm{~m}$ large and $18 \mathrm{~m}$ long, with the beam pipe passing 
through. It will be filled with Neon gas at roughly atmospheric pressure, corresponding to $5.6 \%$ radiation lengths.

In order to have full acceptance coverage for the photons emitted by the decay pions and muons, the total surface of the spherical mirrors must have a diameter of about $3 \mathrm{~m}$. Since the construction of a single spherical mirror of this size is impractical, a mosaic given by 20 hexagonal mirrors made of $2.5 \mathrm{~cm}$ thick glass, each one inscribed inside a $70 \mathrm{~cm}$ diameter circle and with $17 \mathrm{~m}$ focal length, will be used. Two of those mirrors will be obtained from one single hexagonal mirror cut in the middle and with a central hole, in order to fit the central part of the $\mathrm{RICH}$ crossed by the beam pipe containing the primary particle beam.

To avoid the beam pipe shadow on the reflected Cherenkov photons, one half of the mirrors will be oriented towards the right side of the beam pipe and one half towards the left one, thus defining two regions in the focal plane to be equipped with photomultipliers, out of the detector acceptance. The centre of each PM region is about $1 \mathrm{~m}$ far from the beam pipe axis.

The Hamamatsu R7400-U03 photomultipliers have been chosen as light readout device, after test results. They are metal packaged single-anode PM, with UV glass window and cylindrical shape, $16 \mathrm{~mm}$ wide ( $8 \mathrm{~mm}$ minimum active diameter) and $11.5 \mathrm{~mm}$ long. The $\mathrm{R} 7400$ typical rise time is $0.78 \mathrm{~ns}$, the transit time is $5.4 \mathrm{~ns}$ and the transit time jitter is $0.28 \mathrm{~ns}$ (FWHM). The gain measured at $900 \mathrm{~V}$ is about $10^{6}$. The wavelength sensitivity ranges between 185 and $650 \mathrm{~nm}$, with maximum response at $420 \mathrm{~nm}$, where the quantum efficiency is about $20 \%$. In order to convey the Cherenkov light to the active area of the PM, Winston Cones [11] covered with an aluminized mylar foil will be used.
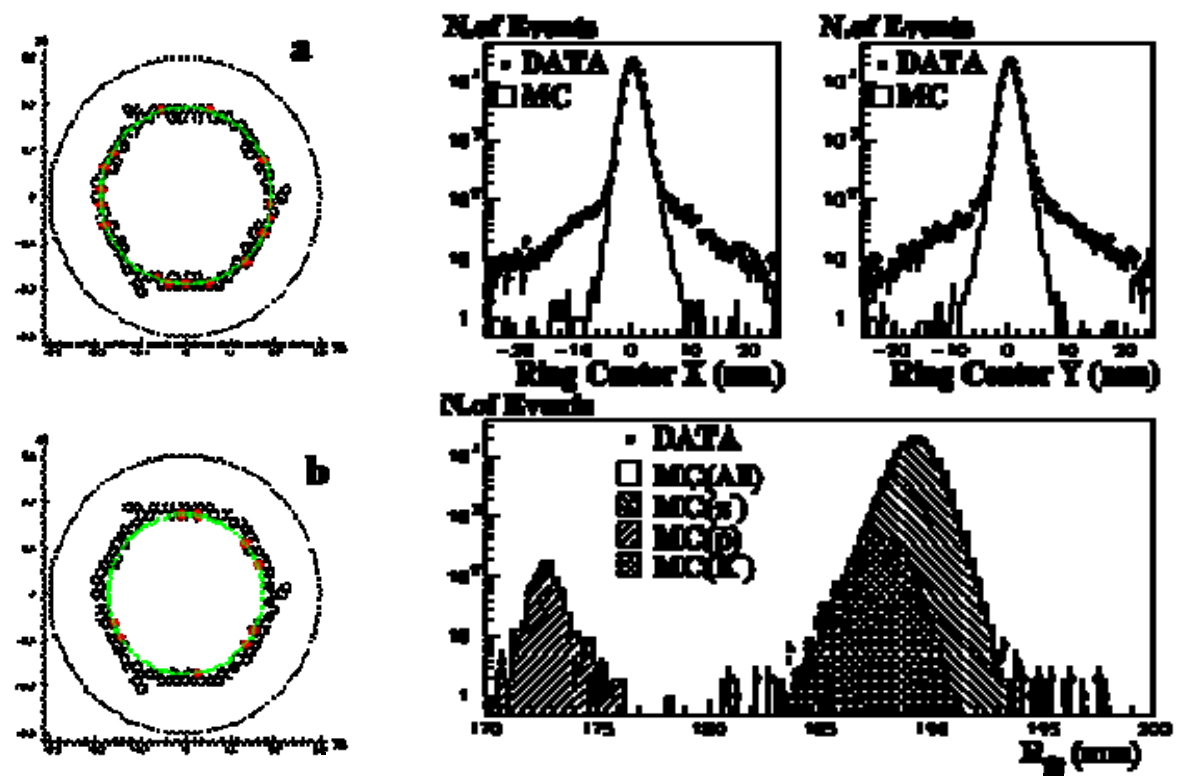

Figure 1: RICH prototype, 2007 beam test results. Left: The ring image for a pion (a) and a proton (b) candidate. Right: Fitted ring center (top) and radius after a $3 \mathrm{~mm}$ cut on the ring center (bottom). 
The PM signal is sent to custom-made current amplifiers with differential output. The amplifiers feed 8-channel NINO ASIC chips [12], used as discriminators operating in timeover-threshold mode. The NINO LVDS output signals are sent to a 512-channel TELL1 board based on 16 HPTDC chips with 97.7 ps LSB [13].

\section{Prototype test results}

A fast simulation was developed taking into account the proper generation of Cherenkov photons, the geometry of the mirror and the PM performances. In order to validate the detector design based on the simulation, a RICH prototype was built and tested.

The RICH prototype [14] consists of a stainless steel vessel made by 5 sections, filled with Neon gas at roughly atmospheric pressure. The two longest sections were recuperated from an existing beam line at CERN. The vessel total length is about $18 \mathrm{~m}$, the diameter is about $60 \mathrm{~cm}$. A single circular $25 \mathrm{~mm}$ thick glass mirror with $50 \mathrm{~cm}$ diameter and $17010 \mathrm{~mm}$ focal length is used, without a beam pipe. The mirror can be moved at high precision by stepping motors, remotely controlled. A laser is used to align the detector with the beam line before the mirror mounting and later used to orient the mirror. The final mirror alignment is done with the beam.

Special care was taken to obtain and keep a good Neon purity inside the vessel and to monitor temperature and pressure during the tests.

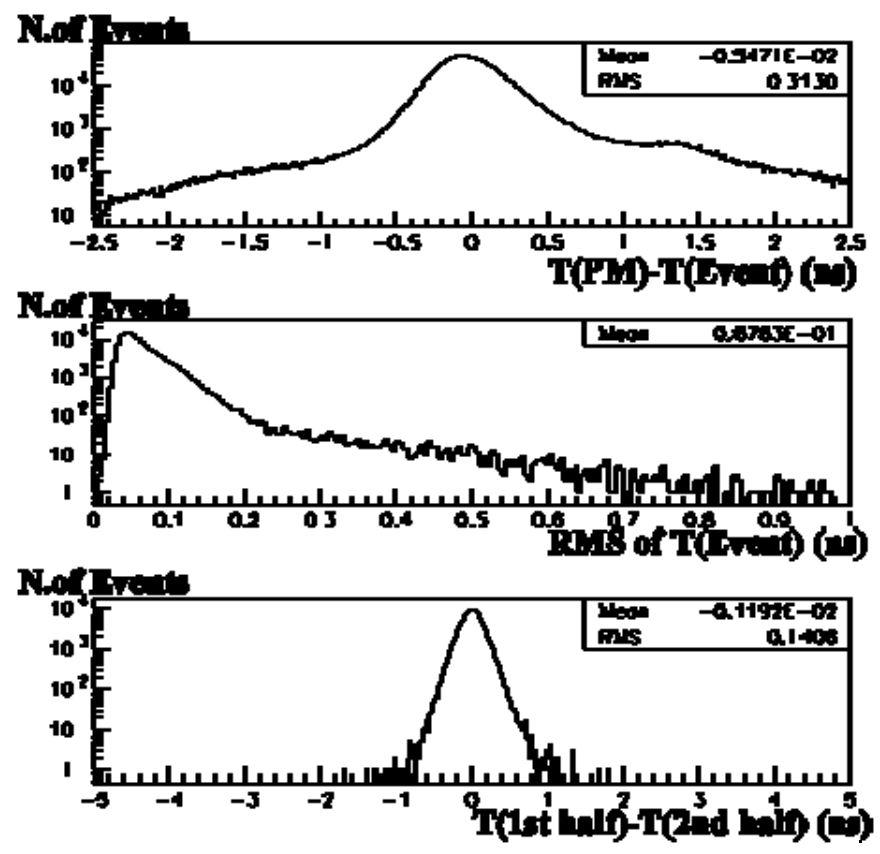

Figure 2: RICH prototype time resolution, beam test 2007 results. Difference between each photomultiplier time and the event time (top); RMS of event time (middle); time difference between the average time of each half of photomultipliers (bottom). 
The 2007 prototype was equipped with 96 photomultipliers. Standard TDC have been used for the time measurement. The results obtained in this former test are in good agreement with the MC expectations, thus validating the choice of the final photomultipliers [14].

An improved prototype with about 400 photomultipliers and a new readout electronics based on the TELL1 boards, similar to the final one, have been tested during late spring in 2009. The cooling system for the photomultipliers and a new mirror have also been tested. The main purpose of this test was to validate the $\pi$ - $\mu$ separation and the final readout electronics design. The results of the data analysis will be published soon.

\subsection{The prototype beam test in 2007}

The RICH prototype was installed at CERN along the K12 beam line and tested for the first time in 2007, with a $200 \mathrm{GeV} / \mathrm{c}$ negative hadron beam (mainly pions) produced by the SPS primary $400 \mathrm{GeV} / \mathrm{c}$ proton beam. Only 96 photomultipliers were installed in the mirror focal plane, along the ring expected for pions at $200 \mathrm{GeV} / \mathrm{c}$ momentum (see Fig. 1, left). A standard VME system with CAEN V1190 TDC modules, based on HPTDC with 100 ps LSB, has been used in this test.

The detector and the analysis of the data for the 2007 test are described in detail in [14]. The performances of the detector in terms of Cherenkov angle resolution, number of photoelectrons and time resolution are in agreement with the Monte Carlo expectations and fully match the detector design.

The average number of PM hits per events was 17 for a pion and 6 for an antiproton, with $30 \%$ probability for a PM to fire if crossed in the center by a Cherenkov ring: the ring images for a pion and an antiproton candidate event are shown in Fig.1, left. If at least four hits were present within $\pm 5 \mathrm{~ns}$, the Cherenkov ring was fitted.

The pion Cherenkov angle resolution was about $50 \mu \mathrm{rad}$ after a $\pm 3 \mathrm{~mm}$ cut on the fitted ring center, shown in Fig.1, rigth-top. The pions and antiproton rings are clearly separated, as also shown in Fig.1 (right-bottom), where the good agreement between data and Monte Carlo predictions is visible.
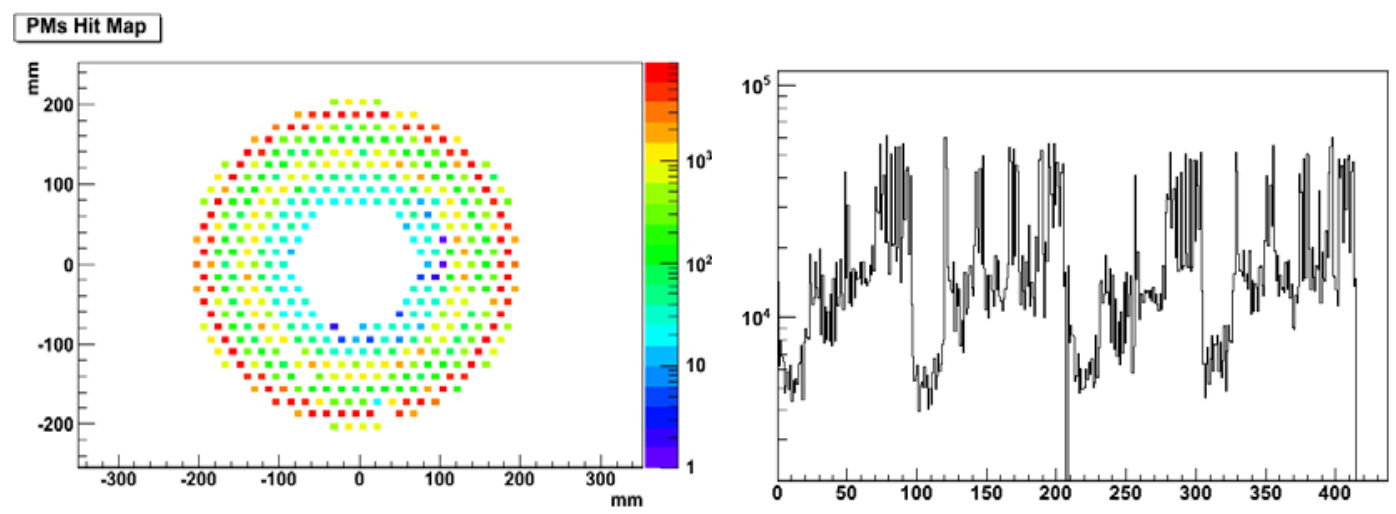

Figure 3: RICH prototype, beam test 2009 preliminary data: PM illumination and hit map. 
The PM signals were properly time aligned and corrected for slewing effects: after applying tight time cuts to the single PM response with respect to the average time, an average single PM time resolution of 310 ps was found (Fig.2, top), while the RMS of the average event time was measured to be 65 ps (Fig.2, middle). As a further check, the difference between the average time of the two half of PM in each event was measured with a resolution of $140 \mathrm{ps,}$ as shown in Fig.2, bottom.

\subsection{The prototype beam test in 2009}

The improved version of the RICH prototype, installed at CERN along the K12 beam line, was tested in may-june 2009. The upstream flange has been arranged in order to accommodate 414 PM. Positive hadron beams of various momenta have been used to check the separation of different particles as a function of the momentum.

The preliminary results of this beam test look very promising. Fig. 3 shows the prototype PM illumination and the hit map after switching on the detector at the beginning of the test: all the channels are properly working; a known missing channel appears in the hit map. The paper reporting the final results of the 2009 beam test is in preparation.

\section{References}

[1] G. Anelli et al., CERN-SPSC-2005-013, CERN-SPSC-P-326 (2005);

G. Ruggiero, in these proceedings.

[2] A.J. Buras, M.Gorbahn, U.Haisch and U.Nierste, JHEP 0611 (2006) 2, HEP-PH 0603079.

[3] G. Isidori, F. Mescia and C. Smith, Nucl. Phys. B 718 (2005) 319.

[4] F. Mescia and C Smith, Phys. Rev. D 76 (2007) 034017.

[5] J. Brod and M. Gorbahn, Phys. Rev. D 78 (2008) 034006.

[6] A.V. Artamonov et al., BNL-E949 Coll., Phys. Rev. D 79 (2009) 092004.

[7] G. Isidori, F .Mescia, P. Paradisi, C. Smith and S. Trine, JHEP 0608 (2006) 064, HEP-PH 0604074.

[8] M. Blanke et al., JHEP 0701 (2007) 066, HEP-PH 0610298.

[9] J. Seguinot and T. Ypsilantis, Nucl. Instr. and Meth. 142 (1977) 377.

[10] A. Bideau-Mehu et al., J. Quant. Spectrosc. Radiat. Transfer 25 (1981) 395.

[11] R. Winston, J. Opt. Soc. Am. 60 (1970) 245.

[12] F. Anghinolfi al., Nucl. Instr. and Meth. A 533 (2004) 183.

[13] G.Haefeli, et al, "TELL1 Specification for a common read out board for LHCb", LHCb 2003-007, IPHE 2003-02;

[14] G. Anzivino et al., Nucl. Instr. and Meth. A 593 (2008) 314. 